\title{
Secreted glycoprotein YKL-40: A potential cancer biomarker and therapeutic target
}

\author{
Rong Shao ${ }^{1-3 *}$ \\ ${ }^{1}$ Department of Pharmacology and Biological Chemistry, Shanghai Jiao Tong University School of Medicine, China \\ ${ }^{2}$ Department of Surgery and Shanghai Key Laboratory of Biliary Tract Disease, Xinhua Hospital, Shanghai Jiao Tong University School of Medicine, China \\ ${ }^{3}$ Department of Biology, University of Massachusetts, USA
}

Over the past two decades, we have substantially gained knowledge about the secreted glycoprotein YKL-40, also named chitinase 3-like 1 (Chi3L1) in many aspects of research field arranging from basic molecular biology to clinical applications. In 1990's, YKL-40 was found to be expressed by cells in a variety of normal tissues including chondrocytes and synoviocytes in bone, vascular smooth muscle cells in blood vessels, and macrophages in the immune system. Thus, its biological and physiological activity is associated with bone-related cell proliferation, vascular cell migration, and macrophage differentiation. Given the evidence that YKL-40 was initially identified from cultured medium of an osteosarcoma line MG-63 cells, it was not unexpected that abundance of subsequent research interest had focused on the aberrant expression of YKL-40 in many cancers and other human disorders. Elevated serum levels of YKL-40 were demonstrated in patients with breast cancer, colorectal cancer, ovarian cancer, leukemia, lymphoma, and glioblastoma. Furthermore, increased YKL-40 serum levels in these cancer patients were correlated with reduced cancer survival and/or cancer metastases [1]. Therefore, YKL-40 has been pointed to be a diagnostic and prognostic biomarker for patients with a broad type of cancers.

Since YKL-40 is a "false" chitinase that lacks hydrolytic activity, even YKL-40 is able to bind to chitin-like oligosaccharides; its pathophysiological activity and molecular mechanisms are not fully understood until recent several years. YKL-40 binds to heparin sulfate chains distributed at ectodomain of membrane protein syndecan-1 [2]. In addition, Dr. J. Elias group has revealed that YKL-40 specifically binds to the membrane receptor IL-13R2 $\alpha$ [3]. A number of research labs including us have reported that intracellular signaling pathways for YKL-40 are associated with activation of FAK-MAPK and PI3K/ Akt, which mediate tumor angiogenesis and tumor cell invasiveness induced by YKL-40. Furthermore, we have recently added large effort to demonstrate that a heparin-binding motif containing a cluster of arginine and lysine residues at $\mathrm{C}$ terminus of YKL-40 is the functional domain that commits YKL-40 to driving angiogenesis [4]. Altogether, these findings have led us to better understand the molecular mechanisms that mediate YKL-40 pathologic activity in multiple human cancers.

Copyright: $(2018$ Shao R. This is an open-access article distributed under the terms of the Creative Commons Attribution License, which permits unrestricted use, distribution, and reproduction in any medium, provided the original author and source are credited.
Albeit these mechanistic insights have offered a number of therapeutic opportunities potential to block YKL-40 function, clinical intervention directly targeting YKL-40 in patients remains to be investigated. It is notable that there are a few pre-clinical trials reported to successfully inhibit YKL-40. For example, YKL-40 gene silence such as shRNA can abrogate tumor growth and angiogenesis [2]. Dr. V. Iragavarapu-Charyulu group employed chitin, a YKL-40 binding ligand, in breast cancer transplanted mice and interestingly found that chitin can protect cancer cells from lung metastasis [5], supporting the notion that competitive binding at YKL-40 heparin-binding motif is an alternative approach to attenuating YKL-40-mediated cancer dissemination. To provide immunotherapeutic value, we recently created a mouse-derived neutralizing antibody against YKL-40 that displays the ability to bind YKL-40 functional domain, thus ablating tumor angiogenesis [4]. These divergent approaches have yielded exciting therapeutic promise arming at YKL-40 in human diseases, not limited to cancers; thereby encouraging us to significantly ameliorate patient's life quality. Hence, powerful tools to potentially block YKL-40 should be urgently taken into account in the next treatment modalities, such as immunotherapy with a humanized antibody and drug delivery systems with nano-particles carrying YKL-40 shRNA or heparinbinding agents.

\section{References}

1. Johansen JS, Schultz NA, Jensen BV (2009) Plasma YKL-40: a potential new cancer biomarker? Future Oncol 5: 1065-1082. [Crossref]

2. Shao R, Hamel K, Petersen L, Cao QJ, Arenas RB, et al. (2009) YKL-40, a secreted glycoprotein, promotes tumor angiogenesis. Oncogene 28: 4456-4468. [Crossref]

3. He CH1, Lee CG, Dela Cruz CS, Lee CM, Zhou Y, et al. (2013) Chitinase 3-like 1 regulates cellular and tissue responses via IL-13 receptor alpha2. Cell Rep 4: 830-841. [Crossref]

4. Ngernyuang N, Yan W, Schwartz LM, Oh D, Liu YB, et al. (2018) A Heparin Binding Motif Rich in Arginine and Lysine is the Functional Domain of YKL-40. Neoplasia 20: 182-192. [Crossref]

5. Libreros S, Iragavarapu-Charyulu V (2015) YKL-40/CHI3L1 drives inflammation on the road of tumor progression. J Leukoc Biol 98: 931-936. [Crossref]
Correspondence to: Rong Shao, Ph.D., Professor, Department of Pharmacology and Biological Chemistry, W. Bldg 3, Rm 407, Shanghai Jiao Tong University School of Medicine, Shanghai, China, 200025, Tel: 021-63846590- Ext: 776497 / 18202115530,E-mail: rongshao@sjtu.edu.cn

Received: February 09, 2018; Accepted: February 24, 2018; Published: February 27,2018 